Communications in Physics, Vol. 27, No. 2 (2017), pp. 165-172

DOI:10.15625/0868-3166/27/2/9633

\title{
INTERACTION BETWEEN TWO CHARGED-HARDCORE SOFT PARTICLES
}

\author{
QUYEN THI LE BUI ${ }^{1}$, THIEN DUC NGO ${ }^{2}$, THUY THI PHUONG NGUYEN ${ }^{2}$ \\ AND HOAI THI LAM NGUYEN $2, \uparrow$ \\ ${ }^{1}$ Thai Binh University of Medicine and Pharmacy, Ly Bon, Thai Binh city \\ ${ }^{2}$ Institute of Physics, Vietnam Academy of Science and Technology, \\ 10 Dao Tan, Ba Dinh, Hanoi, Vietnam \\ ${ }^{\dagger} E$-mail: nlhoai@iop.vast.ac.vn
}

Received 12 April 2017

Accepted for publication 27 July 2017

Published 30 July 2017

\begin{abstract}
During the two last decades, theories of soft-particles have been introduced and developed in order to describe the behavior of bacteria and viruses which can be considered as the bio-colloid particles. Soft particles are assumed to consist of a rigid core inside an ion-permeable shell. The outer layer is always negatively charged while the core can be neutral or charged. In this paper, we investigate the interaction between two identical soft-particles with charged cores. We derived the analytic formula for the interaction energy in a compact form. We showed that the interaction energy involves a screened Coulomb-like interaction term with a distance-independent coefficient. The numerical calculations showed that the interaction energy has very small amplitude and exponentially decays on the distance between the soft particles. The results indicate that it is possible to measure this kind of interaction energy when the system is put into a salt solution with the concentration low enough.
\end{abstract}

Keywords: soft particle, Poisson-Boltzmann equations, electrophoresis mobility, interaction energy.

Classification numbers: 87.10.Ca, 87.15.Pc. 


\section{INTRODUCTION}

Removal of the bacterial and viral pathogens has been always the biggest obstacle in the way of finding out novel water treatment and reuse methods which are low-cost and effective. Many experiments have been conducted for understanding of the factors that have influence on the disinfection process. It is pointed out that the efficiency of the treatment may depend on the interactions among the microbes, on the interaction of the microbes with environmental surfaces, and can be controlled by the water quality parameters such as the $\mathrm{pH}$ and hardness or ionic strength. In order to exploit the mechanism lying behind these behavior properties, theoretical modeling efforts have been made, mostly by H. Oshima $[1,2]$ in his so-called "soft particle electrokinetic theory" (SPE) [2-5]. Here, the protein capsid of the bacteria and virus was described as a softpolyelectrolyte layer which is negatively charged. The word "soft" signifies that it can allow the free-ions and fluid flaws of the aqueous medium in which the particle was immersed to permeate through. This soft layer encloses a hard-core which symbolizes for the part containing the genetic material of the microbial. In SPE, the particle core was of neutral charge and ion nonpenetrable. The model has been successfully applied to investigate the electrophoresis properties of bacteria such as E. coli, S. aureus [6] and MS2 bacteriophage with removed-RNA [7, 8]. By solving the Poisson-Boltzman equation (PBE) and Navier Stock equation for particle motion, the electrophoresis mobility (EPM) of a single soft particle has been derived. The non-zero EPM at infinity ionic strength was predicted. This was a very important contribution of SPE theory which has been proved by experimental data $[9,10]$.

Other authors have contributed on the refinement of SPE theory by modifying the electrostatic structure of the model. It has been shown that either the heterogeneous properties of the soft layer [7,11-13] or the non-neutral charge of the inner core [14-17] would take strong effect on the particle EPM value.

Along with EPM, the data of self-aggregation rate and adhesion efficiency to an environmental surface of microbial system are also important information. These quantities are stipulated by the interaction among the microbial themselves, or between the microbial and the environment surfaces. Therefore, the soft-particles interaction problem is essential in theoretical development. This problem has been studied in Ohshima works, however restricted in simplest limits where the particles are pure colloid (i.e., no soft layer), or pure soft (i.e, zero core-sized) [1]. Interaction in general case, where the soft particles have both charged-core and charged-soft outer layer, has never been mentioned in literature, and thus is the purpose of this works. In this paper, we first derive the expression of interaction energy between two identical soft-particles. We then also perform the numerical calculations for investigating the dependence of interaction energy on the distance between the soft particles and the concentration of salt solution.

The paper is organized as follows. Section II is devoted to the descriptions of the model and the electric potential solution for the Poisson-Boltzmann equation. The analytical and numerical calculations for the interaction energy are also presented and discussed in this section. Conclusions are given in Section III. 


\section{THE INTERACTION BETWEEN TWO SOFT PARTICLES}

\section{II.1. The model}

We present in this section the description of the soft-particle with charged hard core. The model is developed from Ohshima model [3] for the non-treated virus where the bulk RNA is taken into account. Let us discuss on the structure of MS2 bacteriophage [7,8] composed of the bulk RNA, RNA protein-bound, and the protein coat layers. The capsid layer (protein coat) is permeable to the solution and salt ions. The RNA-protein bound prevents water molecules from entering the RNA core. In our model, we supposed that the layer is ion-impermeable to the condition that no more ions flow into the bulk RNA because the core is in a saturated state and hence the volume charge density of the core is reduced to an effective one [8]. We define the "hardcore" to be a combination of the RNA protein bound layer and the bulk RNA. Mathematically, we can assume that the protein bound-layer (cover of the core) is of zero thickness.

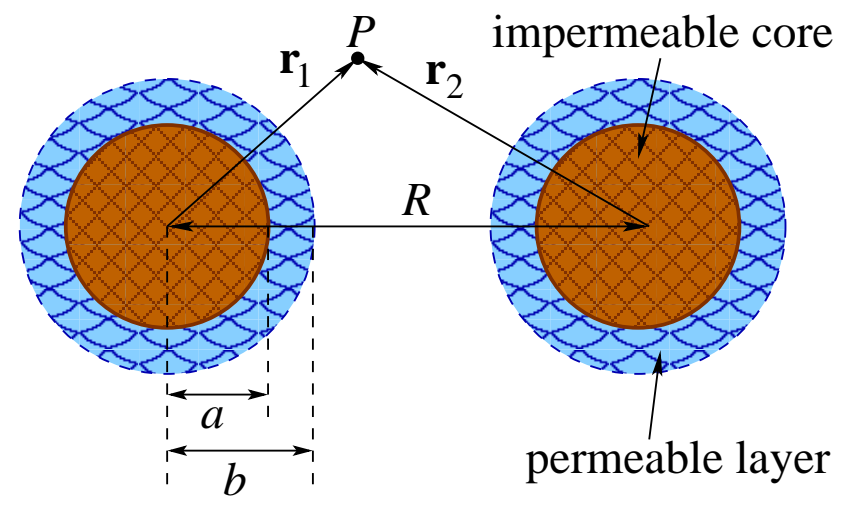

Fig. 1. The core-shell sphere model of two identical soft particles.

Let us consider a system of two identical soft particles separated by distance $R$, labeled by an index $i(i=1,2)$, immersed in a 1:1 electrolyte solution (see in Fig. 1). The soft particle is assumed to contain a spherical hard core of radius $a$ which is surrounded by a concentric shell of polymer-like material (inner radius $a$, outer radius $b$ ). To simplify the presentation we use the subscript notation $c$ and $s$ to denote the core and the shell layer of particle. We denote the uniform charge density of the core and shell layers by $\rho_{c}>0$ and $\rho_{s}=N Z e$ with $N, Z$ and $e$ being the number of atoms per unit volume, the atomic number and the electron charge, respectively.

\section{II.2. Electric potential}

We denote by $\varphi(\boldsymbol{r})$ the electric potential at an arbitrary point in space due to the distributed charges of a soft particle, where $\boldsymbol{r}$ is a vector of magnitude $r$ from the origin (at the center of particle) to the point. This potential can be obtained by solving the Poisson-Boltzmann equation [14]. The general form of this equation in spherical coordinates reads

$$
\frac{d^{2} \varphi(r)}{d r^{2}}+\frac{2}{r} \frac{d \varphi(r)}{d r}=-\frac{\rho_{m}}{\epsilon_{m} \epsilon_{0}}+\kappa^{2} \varphi(r)
$$


with $\rho_{m}$ and $\epsilon_{m}$ being the volume charge density and the relative permittivity of the material, and $\epsilon_{0}$ the permittivity of free space. $\kappa$ is the Debye-Hückel parameter [18] which determines the range of interaction between the particles in the solution and is defined by

$$
\kappa^{2}=\frac{e^{2}}{\epsilon_{r} \epsilon_{0} k_{B} T} \sum_{j=1}^{2} n_{j} z_{j}^{2} \equiv \frac{2 I e^{2}}{\epsilon_{s} \epsilon_{0} k_{B} T}
$$

for 1:1 electrolyte solution such as sodium nitrate, sodium chloride, etc. $I=(1 / 2) \sum_{j=1}^{2}\left(n_{j} z_{j}^{2}\right)$ denotes the ionic strength of the solution with $n_{j}$ and $z_{j}$ being the total number of ions and the charge number of species $j$ in the solution. $k_{B}$ is the Boltzmann constant and $T$ is the temperature. $\epsilon_{s}$ is the relative permittivity of the solution which is assumed to be the same as the one of shell layer of soft particle.

Note that $\kappa=0$ inside the core of particle $(r<a)$ because the mobile ions are only present in the solvent region. In addition, the volume charge density $\rho_{m}$ outside the soft particle $(r>b)$ is equal to zero. Therefore, we can rewrite the Poisson-Boltzmann equation with respect to each region. For convenience, we denote by $\psi_{c}^{(i)}, \psi_{s}^{(i)}$ and $\psi_{o}^{(i)}$ the potentials inside the core, inside the shell and outside the particle $i$. Thus, the equation (1) becomes

$$
\begin{array}{ll}
\frac{d^{2} \psi_{c}^{(i)}(r)}{d r^{2}}+\frac{2}{r} \frac{d \psi_{c}^{(i)}(r)}{d r}=-\frac{\rho_{c}}{\epsilon_{c} \epsilon_{0}} & (r<a), \\
\frac{d^{2} \psi_{s}^{(i)}(r)}{d r^{2}}+\frac{2}{r} \frac{d \psi_{s}^{(i)}(r)}{d r}=-\frac{\rho_{s}}{\epsilon_{s} \epsilon_{0}}+\kappa^{2} \psi_{s}^{(i)}(r) & (a<r<b), \\
\frac{d^{2} \psi_{o}^{(i)}(r)}{d r^{2}}+\frac{2}{r} \frac{d \psi_{o}^{(i)}(r)}{d r}=\kappa^{2} \psi_{o}^{(i)}(r) & (r>a) .
\end{array}
$$

Here $\rho_{c}$ and $\rho_{s}$ are the volume charge density of the core and the shell of soft particle, respectively. The boundary conditions for the potential read

$$
\begin{aligned}
\psi_{c}^{(i)}(a) & =\psi_{s}^{(i)}(a) ; \psi_{s}^{(i)}(b)=\psi_{o}^{(i)}(b), \\
\psi_{c}^{(i)}(0) & \neq 0 ; \psi_{o}^{(i)}(\infty)=0 \\
\left.\epsilon_{c} \epsilon_{0} \frac{d \psi_{c}^{(i)}(r)}{d r}\right|_{r=a} & =\left.\epsilon_{s} \epsilon_{0} \frac{d \psi_{s}^{(i)}(r)}{d r}\right|_{r=a} ;\left.\frac{d \psi_{s}^{(i)}(r)}{d r}\right|_{r=b}=\left.\frac{d \psi_{o}^{(i)}(r)}{d r}\right|_{r=b} .
\end{aligned}
$$

Solving the equations (3)-(5) subject to the boundary conditions (6)-(8), we obtained the solutions for the potential

$$
\begin{aligned}
\psi_{c}^{(i)}(r) & =\frac{\rho_{c}\left(a^{2}-r^{2}\right)}{6 \epsilon_{c} \epsilon_{0}}+\frac{\rho_{c} a^{2}}{3 \epsilon_{s} \epsilon_{0}(1+\kappa a)}+\frac{\rho_{s}}{\epsilon_{s} \epsilon_{0} \kappa^{2}}\left[1-\frac{1+\kappa b}{1+\kappa a} e^{-\kappa(b-a)}\right], \\
\psi_{s}^{(i)}(r) & =\frac{1}{3 \epsilon_{s} \epsilon_{0}} \frac{\rho_{c} a^{3}}{r} \frac{e^{-\kappa(r-a)}}{1+\kappa a} \\
& +\frac{\rho_{s}}{\epsilon_{s} \epsilon_{0}}\left[1-\frac{1+\kappa b}{1+\kappa a}\left(\frac{\sinh \kappa(r-a)}{\kappa r}+\frac{a \cosh \kappa(r-a)}{r}\right)\right]
\end{aligned}
$$


and

$$
\begin{aligned}
\psi_{o}^{(i)}(r) & =\frac{1}{3 \epsilon_{s} \epsilon_{0}} \frac{\rho_{c} a^{3}}{r} \frac{e^{-\kappa(r-a)}}{1+\kappa a} \\
& +\frac{\rho_{s}}{2 \epsilon_{s} \epsilon_{0} \kappa^{2}}\left[1-\frac{1}{\kappa b}+\frac{(1-\kappa a)(1+\kappa b)}{(1+\kappa a) \kappa b} e^{-2 \kappa(b-a)}\right] \frac{b e^{-\kappa(r-b)}}{r}
\end{aligned}
$$

in the regions $(r<a),(a<r<b)$ and $(r>b)$, respectively.

\section{II.3. Interaction energy}

In our model, we assumed that the two soft particles are not overlapped with each other. Because the potential obeys the superposition principle, the total potential of the system at any point $P$ in the space (see in Fig. 1) could be expressed as

$$
\psi(P)=\psi^{(1)}\left(r_{1}\right)+\psi^{(2)}\left(r_{2}\right),
$$

where $r_{i}=\left|\boldsymbol{r}_{i}\right|(i=1,2)$. The interaction energy $[1,14]$ of this system could be obtained by

$$
E(R)=F(R)-F(\infty),
$$

with $F$ being the Helmholtz free energy at a given separation $R>2 b$ and at infinite separation $(R \rightarrow \infty)$ which is given by

$$
\begin{aligned}
F(R) & =\frac{1}{2} \int_{V^{(1)}} \rho_{m}^{(1)}\left[\psi^{(1)}\left(r_{1}\right)+\psi^{(2)}\left(r_{2}\right)\right] d V^{(1)} \\
& +\frac{1}{2} \int_{V^{(2)}} \rho_{m}^{(2)}\left[\psi^{(1)}\left(r_{1}\right)+\psi^{(2)}\left(r_{2}\right)\right] d V^{(2)}
\end{aligned}
$$

and

$$
F(\infty)=\frac{1}{2} \int_{V^{(1)}} \rho_{m}^{(1)} \psi^{(1)}\left(r_{1}\right) d V^{(1)}+\frac{1}{2} \int_{V^{(2)}} \rho_{m}^{(2)} \psi^{(2)}\left(r_{2}\right) d V^{(2)}
$$

in which $\rho_{m}^{(i)}$ and $V^{(i)}$ are the volume charge density and the volume of particle $i$.

Substituting the equations (14) and (15) into (13), we have

$$
E(R)=\frac{1}{2} \int_{V^{(1)}} \rho_{m}^{(1)} \psi^{(2)}\left(r_{2}\right) d V^{(1)}+\frac{1}{2} \int_{V^{(2)}} \rho_{m}^{(2)} \psi^{(1)}\left(r_{1}\right) d V^{(2)}
$$

The distances from the two particles to a given point obey the relation $r_{2}=\left(R^{2}+r_{1}^{2}-2 R r_{1} \cos \theta\right)^{1 / 2}$. For simplicity, we change the notations from $r_{1}$ to $r, r_{2}$ to $r^{\prime}$. The volume element in spherical coordinates is $d V=2 \pi \sin \theta d \theta r^{2} d r$, so the equation (16) can be rewritten in the form

$$
\begin{aligned}
E(R) & =\int_{V} \rho_{m} \psi_{o}\left(r^{\prime}\right) d V \\
& =2 \pi \int_{0}^{\pi} \sin \theta d \theta\left[\int_{0}^{a} \rho_{c} \psi_{o}\left(r^{\prime}\right) r^{2} d r+\int_{a}^{b} \rho_{s} \psi_{o}\left(r^{\prime}\right) r^{2} d r\right] .
\end{aligned}
$$

Integrating Eq. (17) using the formula of potential (11), we have

$$
E(R)=E_{0} \times \frac{e^{-\kappa R}}{\kappa R}
$$


with

$$
\begin{aligned}
E_{0} & =\frac{\pi}{\epsilon_{s} \epsilon_{0}}\left\{\frac{2 a^{4} \rho_{c}\left(\rho_{c}-\rho_{s}\right)}{3 \kappa(1+\kappa a)}\left[1+e^{-2 \kappa a}-\frac{1-e^{-2 \kappa a}}{\kappa a}\right] e^{2 \kappa a}\right. \\
& +\frac{a b \rho_{s}\left(\rho_{c}-\rho_{s}\right)}{\kappa^{3}}\left[1-\frac{1}{\kappa b}+\frac{(1-\kappa a)(1+\kappa b)}{(1+\kappa a) \kappa b} e^{-2 \kappa(b-a)}\right]\left[1+e^{-2 \kappa a}-\frac{1-e^{-2 \kappa a}}{\kappa a}\right] e^{\kappa(b+a)} \\
& +\frac{2 a^{3} b \rho_{c} \rho_{s}}{3 \kappa(1+\kappa a)}\left[1+e^{-2 \kappa b}-\frac{1-e^{-2 \kappa b}}{\kappa b}\right] e^{\kappa(b+a)} \\
& \left.+\frac{b^{2} \rho_{s}^{2}}{\kappa^{3}}\left[1-\frac{1}{\kappa b}+\frac{(1-\kappa a)(1+\kappa b)}{(1+\kappa a) \kappa b} e^{-2 \kappa(b-a)}\right]\left[1+e^{-2 \kappa b}-\frac{1-e^{-2 \kappa b}}{\kappa b}\right] e^{2 \kappa b}\right\}
\end{aligned}
$$

The equations (18) and (19) indicate that the interaction energy has been separated into two terms, one depends on the composition of particle and the other one exponentially decays on the separation $R$. Now, we consider the two limit cases for verifying the analytical calculations.

i) The particles have only the hard core. By setting $\rho_{s}=0$ in Eq. (19), we obtain

$$
E(R)=\frac{2 \pi}{3 \epsilon_{s} \epsilon_{0}} \frac{a^{4} \rho_{c}^{2}}{\kappa(1+\kappa a)}\left(1+e^{-2 \kappa a}-\frac{1-e^{-2 \kappa a}}{\kappa a}\right) \frac{e^{-\kappa(R-2 a)}}{\kappa R} .
$$

Moreover, in the case of salt-free aqueous solution $\kappa \rightarrow 0$, the expression of energy has a well-known form of Coulomb interaction between the two individual charges

$$
E(R) \rightarrow \frac{Q_{\text {core }}^{2}}{4 \pi \epsilon_{s} \epsilon_{0} R}
$$

with $Q_{\text {core }}=4 \pi a^{3} \rho_{c} / 3$ being the total charge in the spherical core of radius $a$.

ii) In the case the particles are without the core $a \rightarrow 0$, the model reduces to a system of two completely ion-permeable particles [1]. So, we should get back the same expression for the interaction energy

$$
E(R)=\frac{\pi}{\epsilon_{s} \epsilon_{0}} \frac{b^{2} \rho_{s}^{2}}{\kappa^{3}}\left(1+e^{-2 \kappa b}-\frac{1-e^{-2 \kappa b}}{\kappa b}\right)^{2} \frac{e^{-\kappa(R-2 b)}}{\kappa R} .
$$

Before closing this section, let us perform the numerical calculation for the interaction energy using the following parameters of the bacteriophage MS2 virus [19]. The viruses consist of an inner RNA component and an outer capsid shell which are corresponding to the core and the shell of the soft particle of radii $a=11.3 \mathrm{~nm}$ and $b=13.4 \mathrm{~nm}$, respectively. For the solution of sodium nitrate $\left(\mathrm{NaNO}_{3}\right)$ in water at room temperature $T=300 \mathrm{~K}$, we take $\epsilon_{s}=78, z_{j}=1$, $n_{j}=1000 N_{a} c$ with $c$ the concentration of ions in units of molar and $N_{a}$ the Avogadro constant. Thus, the Debye length can be evaluated as $\kappa^{-1}=3.04 \times 10^{-10} / \sqrt{c}$, which is expressed in meters. The volume charge densities in the core and in the soft layer are $\rho_{c}=-4.8243 \times 10^{6} \mathrm{C} / \mathrm{mol}$ and $\rho_{s}=-2.4121 \times 10^{6} \mathrm{C} / \mathrm{mol}$. The interaction energy (18) is numerically evaluated and presented in Fig. 2 for several values of salt concentration. It clearly shows that the interaction energy exponentially decreases with increasing the distance between the two soft particles as well as strongly depends on the salt concentration conditions. For example, at the distance $R=30 \mathrm{~nm}$ where the two particles are almost close to each other, the energy is about $2.831 \mathrm{eV}$ for $c=1$ $\mathrm{mM}$ which is ten times greater than that for $c=5 \mathrm{mM}$. Furthermore, the energy is around zero at 


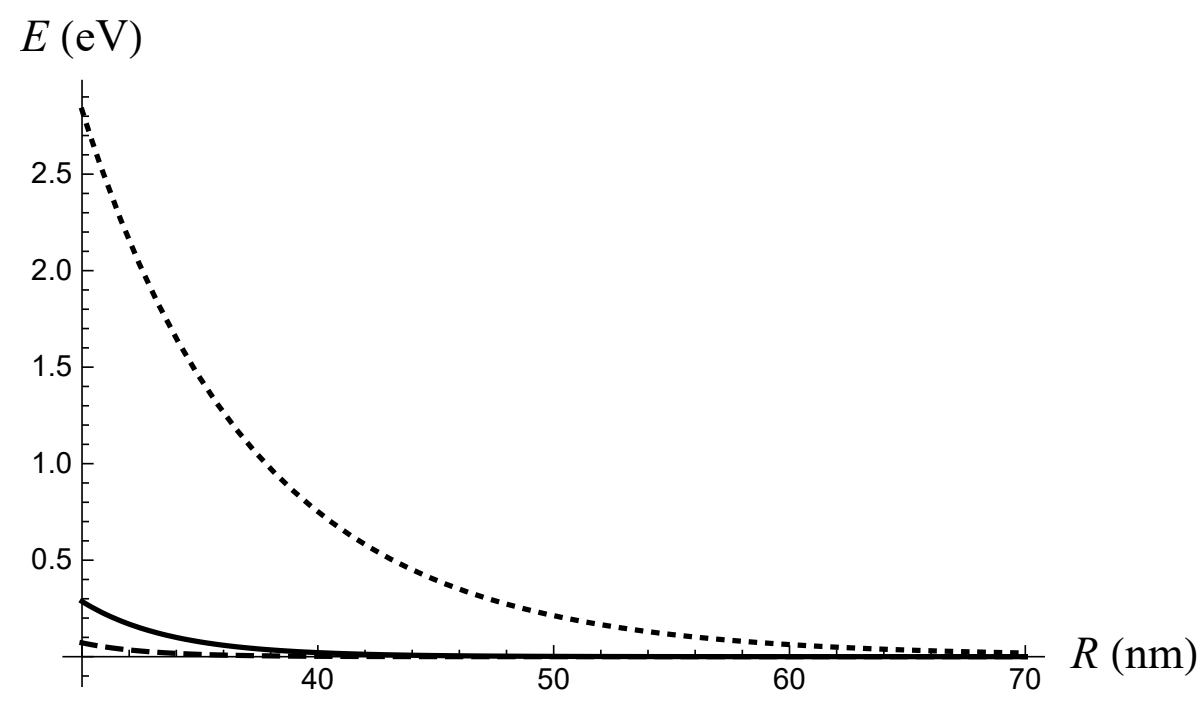

Fig. 2. The interaction energy as a function of the distance between the two soft particles for $1 \mathrm{mM}$ (dotted), $5 \mathrm{mM}$ (solid) and $10 \mathrm{mM}$ (dashed) salt concentration conditions.

any distance $R$ for higher concentration ( $c \geq 10 \mathrm{mM}$ ). These effects could be easily explained as the Coulomb interaction between the charges through an electrostatic screening of salt solution. The solution with a higher ion concentration can cause a stronger screening which reduces the interaction energy.

\section{CONCLUSION}

The interaction between the viruses in a biology system has been demonstrated in a simplest case consisting of the two identical soft particles. From the solution of the set of PoissonBoltzmann equations, we have obtained the analytical expression for the interaction energy of this system. The results are verified by investigating the two limit cases in which the particles are without the shell layer or without the hard core. It is excellent agreement with the previous theory [1].

Our results showed that the Coulomb-like interaction between the soft particles is quite weak. The energy is only a few electronvolt for a system of the packed particles in a low concentration of salt ions. For the experimental measurements, one has to choose the salt concentration conditions around $1.0 \mathrm{mM}$ in order to identify the interaction energy. The results can be also developed for studying more complex models such as the system of the soft particles with different sizes, or a system of the soft and metal particles,... These are the motivations of our future works.

\section{REFERENCES}

[1] H. Ohshima, Theory of colloid and interfacial electric phenomena, 1st ed., Interface Science and Technology 12, Academic Press, 2006.

[2] H. Ohshima, Advances in Colloid and Interface Science 62 (1995) 189-235.

[3] H. Ohshima, Journal of Colloid and Interface Science 163 (1994) 474-483.

[4] H. Ohshima, Sci. Technol. Adv. Mater. 10 (2009) 063001. 
[5] H. Ohshima, Current Opinion in Colloid \& Interface Science 18 (2013) 73-82.

[6] R. Sonohara, N. Muramatsu, H. Ohshima and T. Kondo, Biophysical chemistry 55 (1995) 273-277.

[7] J. Langlet, F. Gaboriaud, C. Gantzer and J. F. L. Duval, Biophys J. 94 (2008) 3293-3312.

[8] T. H. Nguyen, N. Easter, L. Gutierrez, L. Huyett, E. Defnet, S. E. Mylon, J. K. Ferri and A. V. Nguyen, Soft Matter 7 (2011) 10449-10456.

[9] A. J. de Kerchove and M. Elimelech, Langmuir 21 (2005) 6462-6472.

[10] Y. Liu, D. Janjaroen, M. S. Kuhlenschmidt, T. B. Kuhlenschmidt and T. H. Nguyen, Langmuir 25 (2009) 15941605.

[11] J. F. L. Duval and H. Ohshima, Langmuir 22 (2006) 3533-3546.

[12] J. F. L. Duval and F. Gaboriaudb, Current Opinion in Colloid \& Interface Science 15 (2010) 184-195.

[13] J. F. L. Duval, J. Merlin and P. A. L. Narayana, Phys. Chem. Chem. Phys. 13 (2011) 1037-1053.

[14] A. D. Phan, D. A. Tracy, T. L. H. Nguyen, N. A. Viet, T.-L. Phan and T. H. Nguyen, J. Chem. Phys. 139 (2013) 244908.

[15] P. P. Gopmandal, S. Bhattacharyya, M. Banerjee and H. Ohshima, Colloid and Polymer Science 294 (2016) 18451856.

[16] K. McDaniel, F. Valcius, J. Andrews and S. Das, Colloids and Surfaces B: Biointerfaces 127 (2015) 143-147.

[17] S. Bhattacharyya and S. De, Phys. Fluids 28 (2016) 012001.

[18] P. Debye and E. Hückel, Physik. Z. 24 (1923) 185-305.

[19] C. Dika, J. F. L. Duval, H. M. Ly-Chatain, C. Merlin, and C. Gantzer, Applied and Environmental Microbiology 77 (2011) 4939-4948. 